\title{
Principales causas de consulta de urgencias oftalmológicas en un instituto de atención terciaria en Colombia
}

Primary causes of emergency ophthalmological consultations at a tertiary care institution in Colombia

Principais causas de atendimento oftalmológico emergencial em uma instituição de atenção terciária na Colômbia

Virgilio Galvis, MD., Esp., PhD. ${ }^{1}{ }^{\mathbb{O}}$, Alberto Luis Díaz, MD., Esp. ${ }^{2}{ }^{\mathbb{O}}$, Miguel E Ochoa, MD, Msc. ${ }^{3}{ }^{\mathbb{O}}$, Juan José Rey, MD, Msc. ${ }^{3}$, Lizeth C Ardila, MD. ${ }^{4}$ (I) , Lina P Olivero, MD. ${ }^{4}$, Alejandro Tello, MD., Esp., PhD. ${ }^{1}$ it

1. Médico, Especialista en Oftalmología, Subespecialista en Facoemulsificación y Segmento Anterior, Subespecialista en Cirugía refractiva, Doctorado en Ciencias de la Visión, Centro Oftalmológico Virgilio Galvis, Universidad Autónoma de Bucaramanga, Fundación Oftalmológica de Santander, Floridablanca, Santander, Colombia.

2. Médico, Especialista en Oftalmología, Subespecialista en Oculoplástica, Fundación Oftalmológica de Santander, Floridablanca, Santander, Colombia.

3. Médico, Magíster en Epidemiología, Universidad Autónoma de Bucaramanga, Floridablanca, Santander, Colombia.

4. Médico, Universidad Autónoma de Bucaramanga, Bucaramanga, Santander, Colombia.

Correspondencia: Alejandro Tello, Médico, Especialista en Oftalmología, Subespecialista en Facoemulsificación y Segmento Anterior, Subespecialista en Cirugía refractiva, Doctorado en Ciencias de la Visión, Centro Oftalmológico Virgilio Galvis, Universidad Autónoma de Bucaramanga, Fundación Oftalmológica de Santander, Calle 155 \#23-60. Centro Oftalmológico Virgilio Galvis, Torre A, Piso 3, Módulo 7. Floridablanca, Santander, Colombia. E-mail: alejandrotello@gmail.com

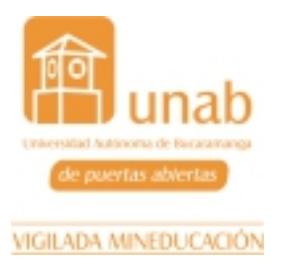

\section{INFORMACIÓN ARTÍCULO}

Artículo recibido: 25 de diciembre de 2018

Artículo aceptado: 29 de julio de 2019

DOI: https://doi.org/10.29375/01237047.3489 
Cómo citar.

Galvis V, Díaz AL, Ochoa ME, Rey JJ, Ardila LC, Olivero LP,

Tello A. Principales causas de consulta de urgencias

oftalmológicas en un

instituto de atención terciaria

en Colombia. MedUNAB

2019;22(2):153-161.

doi:10.29375/01237047.3489

\section{RESUMEN}

Introducción. Diversas condiciones oftalmológicas son causas de consultas a los servicios de urgencias. El objetivo de este trabajo fue determinar las causas más comunes de consulta al servicio de urgencias oftalmológicas de una institución terciaria en Colombia y, así, poder explorar en el futuro la posibilidad de identificar algunas medidas preventivas dirigidas a disminuir la frecuencia de esas causas.

Metodología. Estudio descriptivo retrospectivo basado en los registros de pacientes que asistieron al servicio de urgencias durante los años 2014 y 2015. Las patologías se identificaron a partir de los códigos de la Clasificación Internacional de Enfermedades, CIE-10.

Resultados. Se identificaron 9,088 y 8,162 registros para los años 2014 y 2015, respectivamente. Las causas de consulta a urgencias oftalmológicas más frecuentes para el año 2014 fueron: cuerpo extraño en la córnea o en la conjuntiva con un $20.2 \%$ de los casos, conjuntivitis aguda no especificada con $11.3 \%$, traumatismo de la conjuntiva y abrasión corneal con $8.3 \%$, hemorragia conjuntival con $5.8 \%$ y quemaduras corneales con $4.7 \%$. En el año 2015, las causas fueron: cuerpo extraño en la córnea o en la conjuntiva (23.6\%), traumatismo de la conjuntiva y abrasión corneal $(9.0 \%)$, conjuntivitis aguda no especificada (8.9\%), hemorragia conjuntival $(4.4 \%)$ y quemaduras corneales $(4.0 \%)$.

Discusión. Las causas más frecuentes de consulta a urgencias oftalmológicas incluyeron los traumas y las infecciones del segmento anterior del ojo.

Conclusiones. Existe la posibilidad de identificar algunas medidas preventivas (elementos de protección adecuados, protocolos de conducta en situaciones de riesgo, etc.) que ayuden a disminuir la frecuencia de traumas oculares y minimicen el riesgo de infecciones. Hay, entonces, cabida para más estudios en los que se analicen los mecanismos específicos de estas lesiones.

Palabras clave:

Urgencias Médicas; Oftalmología; Quemaduras Oculares; Enfermedades de la Córnea; Lesiones Oculares; Cuerpos Extraños en el Ojo; Conjuntivitis.

\section{ABSTRACT}

Introduction. Several ophthalmological conditions are causes of consultations to the emergency services. The objective of this study was to determine the most common causes of consultation to the ophthalmological emergency service of a tertiary institution in Colombia and thus be able to explore in the future the possibility of identifying some preventive measures aimed at reducing the frequency of these causes.

Methodology. Retrospective descriptive study based on the records of patients attending the emergency department during 2014 and 2015, identifying the diseases based on the International Classification of Diseases (ICD-10) codes.

Results. 9,088 and 8,162 records were identified for 2014 and 2015, respectively. The most frequent causes of ophthalmologic urgency consultation for the year 2014 were: corneal or conjunctive foreign body with $20.2 \%$, acute conjunctivitis not specified $11.3 \%$, conjunctival trauma and corneal abrasion $8.3 \%$, conjunctival haemorrhage $5.8 \%$ and corneal burns $4.7 \%$. In 2015 the causes were: foreign body in the cornea or conjunctiva $(23.6 \%)$, conjunctival trauma and corneal abrasion $(9.0 \%)$, acute unspecified conjunctivitis $(8.9 \%)$, conjunctival hemorrhage $(4.4 \%)$, and corneal burns $(4.0 \%)$.

Discussion. The most frequent causes of consultation with ophthalmologic emergencies included trauma and infections of the anterior segment of the eye.

Conclusions. There is a possibility of identifying some preventive measures (adequate protection elements, behavior protocols in situations of risk, etc.) that help 
to reduce the frequency of eye traumas and minimize the risk of infections. There is room for more studies in which the specific mechanisms of these injuries are analyzed.

Key words:

Emergencies; Ophthalmology; Eye Burns; Corneal Diseases; Eye Injuries; Eye Foreign Bodies; Conjunctivitis.

\section{RESUMO}

Introdução. Várias condições oftalmológicas são causas de atendimento nos serviços de emergência. $\mathrm{O}$ objetivo deste trabalho foi determinar as causas mais comuns de atendimento oftalmológico emergencial em uma instituição terciária na Colômbia e, assim, poder explorar no futuro a possibilidade de identificar algumas medidas preventivas para reduzir a frequência dessas causas.

Métodos. Estudo descritivo retrospectivo baseado nos registros de pacientes que compareceram ao serviço de emergência oftalmológica durante os anos 2014 e 2015. As patologias foram identificadas a partir dos códigos da Classificação Internacional de Doenças, CID-10.

Resultados. Foram identificados 9,088 e 8,162 registros para os anos 2014 e 2015, respectivamente. As causas de atendimento oftalmológico emergencial mais frequentes em 2014 foram: corpo estranho na córnea ou na conjuntiva com 20,2 \% dos casos, conjuntivite aguda não especificada com 11,3 \%, trauma conjuntival e abrasão corneana com $8,3 \%$, hemorragia conjuntival com $5,8 \%$ e queimaduras corneanas com $4,7 \%$. No ano 2015, as causas foram: corpo estranho na córnea ou na conjuntiva $(23,6 \%)$, trauma conjuntival e abrasão corneana $(9,0 \%)$, conjuntivite aguda não especificada $(8,9 \%)$, hemorragia conjuntival $(4,4 \%)$ e queimaduras corneanas $(4,0 \%)$.

Discussão. As causas mais frequentes de atendimento oftalmológico emergencial incluíram traumas e infecções do segmento anterior do olho.

Conclusões. Existe a possibilidade de identificar algumas medidas preventivas (elementos de proteção adequados, protocolos de conduta em situações de risco, etc.) que ajudem na redução da frequência de trauma ocular e para minimizar o risco de infecções. Há, então, espaço para mais estudos que analisem os mecanismos específicos dessas lesões

Palavras-chave: Emergências; Oftalmologia; Queimaduras Oculares; Doenças da Córnea; Traumatismos Oculares; Corpos Estranhos no Olho; Conjuntivite.

\section{Introducción}

Los pacientes con condiciones oftalmológicas consultan los servicios de urgencias ya sea por lesiones traumáticas o por diversas enfermedades con síntomas variados que, usualmente, afectan la visión o se acompañan de dolor (1-6). Se ha calculado que la tasa de emergencias oftalmológicas se ubica, aproximadamente, entre el $1.2 \%$ y el $6 \%$ de todas las consultas de un servicio de emergencias general $(1,2,7,8)$.

Este estudio se realizó mediante un análisis de los registros electrónicos, con el fin de establecer las causas de consulta de urgencias en el servicio de oftalmología de una institución de atención terciaria en Floridablanca, Colombia. En este país no se encontraron estudios epidemiológicos sobre las principales patologías en urgencias oftalmológicas. La información obtenida permitirá explorar la posibilidad de diseñar, en el futuro, algunas estrategias preventivas para disminuir la ocurrencia de estos eventos.

\section{Metodología}

Estudio descriptivo retrospectivo basado en los registros de pacientes que asistieron al servicio de 
urgencias en la institución seleccionada durante los años 2014 y 2015. Las patologías se identificaron con base en los códigos de la Clasificación Internacional de Enfermedades, CIE-10. Para ello, se revisó el sistema de historia clínica electrónica y se exportaron los datos a una hoja de cálculo en un software informático (Excel ${ }^{\circledR}$ de Microsoft Corporation, Redmond, Washington, EEUU). Se consideró, como criterio de inclusión, haber consultado al servicio de urgencias oftalmológicas luego de una evaluación adecuada por el servicio de triaje. No se consideraron criterios de exclusión. Se tomaron los datos de los 20 primeros diagnósticos registrados.

Este estudio fue considerado sin riesgo y fue aprobado por el Comité de Ética Institucional. Los análisis estadísticos se realizaron utilizando el programa estadístico Stata (versión 14), desarrollado por
Statacorp LLC (College Station, Texas, EEUU).

\section{Resultados}

Se identificaron 9,088 y 8,162 registros para los años 2014 y 2015, respectivamente.

Las primeras veinte causas encontradas en 2014 y 2015 representaron el $70 \%$ de las consultas oftalmológicas al servicio de urgencias.

En las Tablas 1 y 2 se encuentran relacionadas las veinte primeras causas de consulta a urgencias oftalmológicas identificadas en cada uno de los dos años analizados. En la Figura 1 se encuentran las nueve primeras causas de consulta de los dos años que, aunque cambiaron algo en el orden de prevalencia, fueron las mismas.

Tabla 1. Principales causas de consulta a urgencias oftalmológicas año 2014

\begin{tabular}{|c|c|c|c|c|c|}
\hline Orden & Código & Diagnóstico & $\begin{array}{l}\text { Número } \\
\text { de casos }\end{array}$ & Porcentaje & $\begin{array}{l}\text { Porcentaje } \\
\text { acumulado }\end{array}$ \\
\hline 1 & $\mathrm{~T} 150$ & Cuerpo extraño en la córnea & 1,286 & $14.2 \%$ & $14.2 \%$ \\
\hline 2 & $\mathrm{H} 103$ & Conjuntivitis aguda & 1,024 & $11.3 \%$ & $25.4 \%$ \\
\hline 3 & S050 & $\begin{array}{c}\text { Traumatismo de la conjuntiva } \\
\text { y abrasión corneal }\end{array}$ & 754 & $8.3 \%$ & $33.7 \%$ \\
\hline 4 & $\mathrm{~T} 151$ & $\begin{array}{c}\text { Cuerpo extraño en el saco } \\
\text { conjuntival }\end{array}$ & 548 & $6.0 \%$ & $39.7 \%$ \\
\hline 5 & $\mathrm{H} 113$ & Hemorragia conjuntival & 527 & $5.8 \%$ & $45.5 \%$ \\
\hline 6 & $\mathrm{~T} 261$ & $\begin{array}{l}\text { Quemadura de la córnea } \\
\text { y saco conjuntival }\end{array}$ & 425 & $4.7 \%$ & $50.2 \%$ \\
\hline 7 & H000 & Orzuelo & 279 & $3.1 \%$ & $53.3 \%$ \\
\hline 8 & $\mathrm{H} 160$ & Úlcera de la córnea & 274 & $3.0 \%$ & $56.3 \%$ \\
\hline 9 & S051 & $\begin{array}{c}\text { Contusión del globo ocular } \\
\text { y del tejido orbitario }\end{array}$ & 268 & $2.9 \%$ & $59.3 \%$ \\
\hline 10 & Z010 & Examen de ojos y de la visión & 161 & $1.8 \%$ & $61.0 \%$ \\
\hline 11 & H049 & Trastorno del aparato lagrimal & 160 & $1.8 \%$ & $62.8 \%$ \\
\hline 12 & $\mathrm{H} 200$ & Iridociclitis aguda y subaguda & 160 & $1.8 \%$ & $64.5 \%$ \\
\hline 13 & $\mathrm{H} 101$ & Conjuntivitis atópica aguda & 150 & $1.7 \%$ & $66.2 \%$ \\
\hline 14 & $\mathrm{H} 168$ & Otras queratitis & 127 & $1.4 \%$ & $67.6 \%$ \\
\hline
\end{tabular}




\begin{tabular}{|c|c|c|c|c|c|}
\hline 15 & H169 & Queratitis, no especificada & 124 & $1.4 \%$ & $69.0 \%$ \\
\hline 16 & H041 & $\begin{array}{c}\text { Otros trastornos de la glándula } \\
\text { lagrimal }\end{array}$ & 118 & $1.3 \%$ & $70.3 \%$ \\
\hline 17 & $\mathrm{H} 118$ & $\begin{array}{c}\text { Otros trastornos especificados } \\
\text { de la conjuntiva }\end{array}$ & 110 & $1.2 \%$ & $71.5 \%$ \\
\hline 18 & H109 & Conjuntivitis no especificada & 99 & $1.1 \%$ & $72.6 \%$ \\
\hline 19 & $\mathrm{H} 438$ & $\begin{array}{c}\text { Otros trastornos del cuerpo } \\
\text { vítreo }\end{array}$ & 94 & $1.0 \%$ & $73.6 \%$ \\
\hline 20 & H571 & Dolor ocular & 82 & $0.9 \%$ & $74.5 \%$ \\
\hline
\end{tabular}

Fuente: Elaborada por los autores.

Tabla 2. Principales causas de consulta a urgencias oftalmológicas año 2015

\begin{tabular}{|c|c|c|c|c|c|}
\hline Orden & Código & Diagnóstico & $\begin{array}{l}\text { Número } \\
\text { de casos }\end{array}$ & Porcentaje & $\begin{array}{l}\text { Porcentaje } \\
\text { acumulado }\end{array}$ \\
\hline 1 & & Cuerpo extraño en la córnea & 1,199 & $14.7 \%$ & $14.7 \%$ \\
\hline 2 & & $\begin{array}{l}\text { Traumatismo de la conjuntiva, } \\
\text { abrasión corneal }\end{array}$ & 734 & $9.0 \%$ & $23.7 \%$ \\
\hline 3 & & Conjuntivitis aguda & 725 & $8.9 \%$ & $32.6 \%$ \\
\hline 4 & & $\begin{array}{l}\text { Cuerpo extraño en el saco } \\
\text { conjuntival }\end{array}$ & 443 & $5.4 \%$ & $38.0 \%$ \\
\hline 5 & & Hemorragia conjuntival & 357 & $4.4 \%$ & $42.4 \%$ \\
\hline 6 & & $\begin{array}{l}\text { Quemadura de la córnea y saco } \\
\text { conjuntival }\end{array}$ & 323 & $4.0 \%$ & $46.3 \%$ \\
\hline 7 & & Úlcera de la córnea & 240 & $2.9 \%$ & $49.3 \%$ \\
\hline 8 & & Contusión del globo ocular & 230 & $2.8 \%$ & $52.1 \%$ \\
\hline 9 & & Orzuelo & 216 & $2.6 \%$ & $54.7 \%$ \\
\hline 10 & & Iridociclitis aguda y subaguda & 209 & $2.6 \%$ & $57.3 \%$ \\
\hline 11 & & Queratitis no especificada & 147 & $1.8 \%$ & $59.1 \%$ \\
\hline 12 & & $\begin{array}{c}\text { Trastorno del aparato lagrimal no } \\
\text { especificado }\end{array}$ & 132 & $1.6 \%$ & $60.7 \%$ \\
\hline 13 & & $\begin{array}{c}\text { Trastorno de la conjuntiva, no } \\
\text { especificado }\end{array}$ & 111 & $1.4 \%$ & $62.1 \%$ \\
\hline 14 & & Examen de ojos y de la visión & 100 & $1.2 \%$ & $63.3 \%$ \\
\hline 15 & & Otros trastornos del cuerpo vítreo & 97 & $1.2 \%$ & $64.5 \%$ \\
\hline
\end{tabular}


UNAB Principales causas de consulta de urgencias oftalmológicas en un instituto de atención terciaria en Colombia

\begin{tabular}{ccccc}
\hline 16 & $\begin{array}{c}\text { Convalecencia consecutiva a } \\
\text { cirugía }\end{array}$ & 93 & $1.1 \%$ & $65.6 \%$ \\
\hline 17 & Conjuntivitis atópica aguda & 92 & $1.1 \%$ & $66.7 \%$ \\
\hline 18 & Conjuntivitis mucopurulenta & 86 & $1.1 \%$ & $67.8 \%$ \\
\hline 19 & $\begin{array}{c}\text { Otros trastornos específicos } \\
\text { de la conjuntiva }\end{array}$ & 79 & $1.0 \%$ & $68.8 \%$ \\
\hline 20 & Hemorragia del vítreo & 77 & $0.9 \%$ & $69.7 \%$ \\
\hline
\end{tabular}

Fuente: Elaborada por los autores.

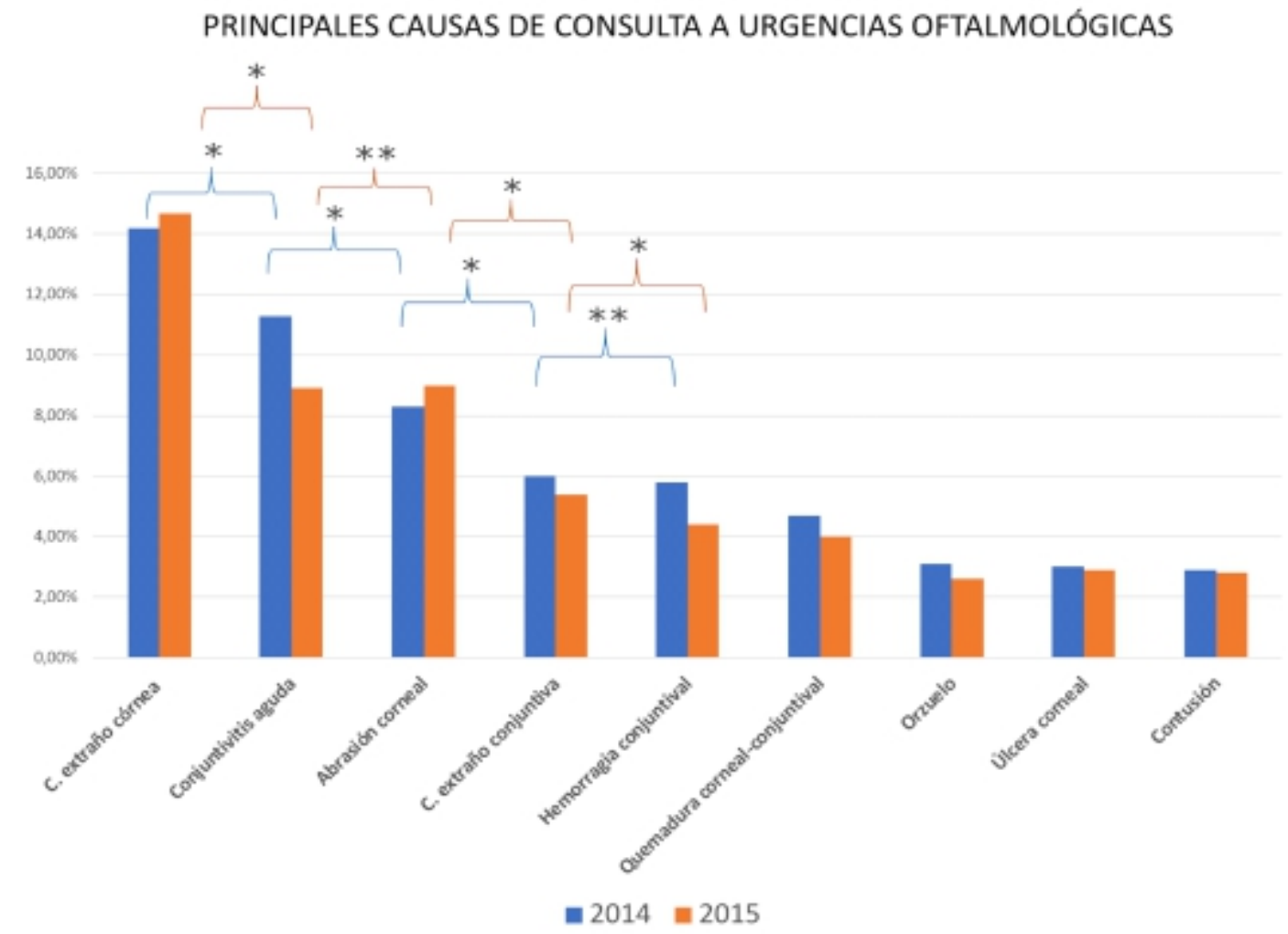

Figura 1. Causas más comunes de consulta oftalmológica de urgencia (2014-2015).

$*=$ Diferencia significativa. $* *=$ Diferencia no significativa. Nivel de significación $\mathrm{p}<0.05$

Fuente: Elaborada por los autores.

Se encontró que, en el año 2014, las causas traumáticas correspondieron al $36.1 \%$ de las consultas y en el 2015 al $35.9 \%$.

\section{Discusión}

El presente estudio se realizó en una institución oftalmológica de tercer nivel ubicada en Floridablanca, una ciudad del nororiente de Colombia, en el departamento de Santander. Ésta atiende directamente a una población de algo más de un millón de habitantes (área metropolitana de Bucaramanga), pero su zona de influencia se extiende, aproximadamente, a cuatro millones de habitantes. Las tres primeras causas de consulta identificadas fueron las mismas en los dos periodos anuales estudiados. El diagnóstico que permaneció en el primer puesto para los dos años analizados (2014 y 2015) fue el de cuerpo extraño en la 
córnea. La conjuntivitis aguda pasó de estar en el segundo puesto en 2014 a ubicarse en el tercer lugar en 2015. Por su parte, el diagnóstico de traumatismo en la córnea-abrasión corneal pasó del tercer lugar a ubicarse en el segundo puesto en 2015 (Tablas 1 y 2).

En Colombia no se encontraron estudios epidemiológicos sobre las principales patologías en urgencias oftalmológicas.

Los hallazgos del presente estudio se correlacionan con el mayor estudio sobre este tema, publicado en 2016 por Channa y coautores (5). Analizando las consultas a los servicios de urgencias relacionadas con afecciones oculares a lo largo de todos los Estados Unidos en un periodo de 6 años (casi 12 millones de casos), estos investigadores identificaron que el diagnóstico más común fue conjuntivitis $(28.0 \%)$, seguido de abrasiones corneales (13.7\%) y cuerpo extraño corneal $(7.5 \%)$. Fueron similares los resultados de un estudio retrospectivo realizado en España, en el cual compararon las consultas de los años 1997 y 2005 . Leal y coautores encontraron que las tres principales causas de consulta a urgencias oftalmológicas, para 1997, fueron problemas corneales $(27.80 \%)$, conjuntivitis $(19.60 \%)$ y cuerpo extraño corneal (13.10\%); en el 2005, los porcentajes fueron cuerpo extraño corneal (39\%), problemas corneales $(17 \%)$ y conjuntivitis $(7.30 \%)(3)$. Estas mismas aparecen como las tres primeras causas de consulta, aunque con una variación en su orden, en estudios realizados en Rumania y Turquía, publicados recientemente (en 2016 y 2018, respectivamente) $(6,9)$.

Por otra parte, encontramos diferencias con los diagnósticos más frecuentes en servicios de urgencia oftalmológicos identificados en otros estudios de diferentes países. En el estudio retrospectivo de 2005 realizado en Australia por Kumar y coautores en un centro de urgencias oftalmológicas, se encontró que las cinco principales causas de consulta fueron: conjuntivitis, queratitis, cataratas, abrasión corneal e iridociclitis. Diagnósticos como conjuntivitis y abrasión corneal fueron también unos de las más frecuentes en el presente estudio. Por el contrario, no se encontró la catarata dentro de las 10 causas más comunes de consulta de urgencia identificadas en la institución. Esto se debe a que los pacientes son clasificados en la evaluación previa antes de ingresar al servicio de urgencias para establecer prioridades (triaje) y la mayoría de los casos no urgentes (como las cataratas) son redirigidos al servicio de consulta externa. Es posible que los pacientes reseñados en el estudio de Kumar y coautores fueran atendidos en el mismo servicio de urgencias, lo que explicaría su alto porcentaje (4). Un fenómeno similar se observó en el estudio publicado en 2019 por Domínguez-Serrano y coautores, realizado en dos hospitales de tercer nivel de España. Aunque las conjuntivitis y las queratitis ocuparon los dos primeros lugares, la hemorragia subconjuntival (hiposfagma) y el desprendimiento de vítreo posterior se ubicaron en los siguientes dos lugares, desplazando al cuerpo extraño a una quinta posición. Dentro de las diez primeras causas de consulta de urgencias por condiciones oculares, estos investigadores identificaron también la blefaritis y el ojo seco. Ellos, analizando tal situación, concluyeron que un porcentaje muy importante de las condiciones oculares atendidas en el servicio de urgencias, en realidad, eran no urgentes $(73.4 \%)$ y sugirieron, en consecuencia, que se establecieran medidas en España para conseguir una asistencia más eficiente, orientada a la patología urgente y que no consumiese recursos innecesarios (10). En los últimos años, los servicios de atención de urgencias oftalmológicas en el Reino Unido y otros países (como Nueva Zelanda) han mostrado un gran aumento en el número de consultas y también se ha visto, al igual que en España, que un importante porcentaje de estas son, en realidad, problemas no urgentes que podrían tratarse en servicios de consulta externa $(11,12)$. Hallazgos similares, aunque con porcentajes menos elevados, han sido recientemente publicados en los Estados Unidos. Un estudio nacional (con una muestra de más de 11 millones de casos) mostró que casi el $25 \%$ de una población de adultos que habían asistido a una consulta en el servicio de urgencias por alguna condición oftalmológica había recibido el diagnóstico de una condición no urgente (incluyendo como no urgente las conjuntivitis) (13). Otro estudio, previamente ya citado, también de carácter nacional en ese país, que analizó casi 12 millones de consultas de urgencias por condiciones oftalmológicas, encontró que, aún incluyendo las conjuntivitis como una urgencia, el $30.8 \%$ de las consultas a urgencias identificadas en todos los Estados Unidos no eran condiciones que requerían la atención en ese departamento y hubiesen podido ser referidas a otra dependencia (5). El mecanismo de triaje que se usa en nuestra institución, establecido en casi todos los servicios de urgencias en Colombia, controla este tipo de fenómenos que pueden llegar a consumir más recursos del sistema de salud de manera no óptima. El filtro consiste en la evaluación de la pertinencia de la consulta antes del ingreso; si no hay una condición urgente, se direcciona al paciente a un servicio de consulta externa. 
A finales de la década de 1980, en el Reino Unido, Edwards encontró que el trauma constituía un $65.6 \%$ de las causas de consulta oftalmológica en un servicio de urgencias (14). De manera similar, en 2002, en Francia, Girard y coautores reportaron que la primera causa de consulta oftalmológica de urgencia se relacionó con lesiones traumáticas en aproximadamente un $66 \%$ de los casos (1). Estos porcentajes son mayores que el encontrado en el presente estudio (que estuvo alrededor de un $36 \%$ ). Por otra parte, en 2016, Channa y coautores en su estudio nacional en los Estados Unidos reportaron un valor muy similar al encontrado en el presente estudio (36.6 \%) (5). Vieira, en Brasil, en 2008, publicó que los cuerpos extraños correspondieron a un $20 \%$ y otros traumas oculares a un $10 \%$ de los casos de urgencia (15). Similarmente, un estudio publicado en 2018 indicó que en Francia el 26.1 \% de las emergencias oculares correspondieron a trauma (16). Otros estudios han encontrado porcentajes algo menores: en Arabia Saudita, en el año 2014, el trauma ocular fue la causa de consulta solo en el $19.1 \%$ de los casos (17).

En cuanto a las circunstancias de origen de los traumas oculares, en España, a mediados de la década de 1990, León y coautores encontraron que los accidentes laborales ocupaban el primer lugar (21.9\%), seguidos por los accidentes domésticos $(21 \%)$, los accidentes en tiempo de ocio (17\%), los accidentes automovilísticos $(15 \%)$, los accidentes en actividades deportivas (12\%) y los traumas por agresión o asalto (10\%) (18). Fea y coautores, en 2008, publicaron sus resultados acerca de un estudio sobre trauma ocular en Italia. La mayoría de los traumas ocurrieron en el trabajo $(57.12 \%)$ o en el hogar $(35.15 \%)$. Los cuerpos extraños corneales fueron la primera causa de consulta $(49.5 \%)$, seguidos por las abrasiones corneales $(29.6 \%)$ y las abrasiones conjuntivales (16.5\%) (19). Los traumas relacionados con deportes pueden causar severas lesiones oculares y, en general, las medidas preventivas son muy efectivas (20). En Irán, Jafari y coautores encontraron que los traumas relacionados con actividades laborales fueron la causa más común de consulta en un servicio de urgencias oftalmológicas (30.3\%); dentro de estos, las esquirlas metálicas ocuparon el primer lugar. Solo el $18.6 \%$ de los pacientes con trauma ocular usaba gafas de protección (7).

En un estudio en Líbano encontraron una disminución de las lesiones oculares penetrantes entre los años 1997 y 2012, y los autores atribuyeron este fenómeno a las campañas de salud pública desarrolladas en cuanto a elementos de seguridad al conducir y en las obras de construcción, lo cual sugiere un efecto benéfico de este tipo de acciones (2).

En la cohorte incluida en el presente estudio, entre el $4.0 \%$ y el $4.7 \%$ de las consultas de urgencia se relacionó con quemaduras de la superficie ocular. No hay muchos estudios que reporten las proporciones de la frecuencia de las quemaduras de córnea y conjuntiva con respecto al total de urgencias oftalmológicas y sus resultados son variables. Mientras que Girard y coautores, en Francia, reportaron que el $6.4 \%$ de las urgencias oftálmicas se relacionaron con quemaduras, recientemente, otros grupos de investigadores encontraron porcentajes menores: Samoilă y coautores en Rumania un $1.3 \%$ y, en el estudio nacional de los Estados Unidos, Channa y coautores encontraron un $0.9 \%(1,5,9)$.

Con el fin de obtener información más detallada de los registros clasificados de acuerdo a los códigos del CIE 10 , se deben parametrizar clasificaciones adicionales más específicas, que estén a disposición del médico tratante al momento de definir el diagnóstico.

Una debilidad del presente estudio es que no se recolectaron datos adicionales en cuanto a los mecanismos y las circunstancias específicas de las lesiones traumáticas. Adicionalmente, el diseño retrospectivo hace que variables importantes (que pudieron haberse analizado) no se recogieran de manera apropiada. Es importante que tanto oftalmólogos como pacientes se esfuercen por mejorar la prevención de las principales patologías oculares que llevan a consultar al servicio de urgencias, ya que muchas de ellas son causa importante de discapacidad parcial o total.

\section{Conclusiones}

Se identificó que las causas más frecuentes de consulta a urgencias oftalmológicas en una institución oftalmológica de atención terciaria en Colombia incluyeron, en orden de frecuencia, los traumas corneales y las infecciones del segmento anterior del ojo. La córnea y la conjuntiva ocuparon los primeros puestos como lugares anatómicos comprometidos.

Se requiere realizar ajustes al programa de captura de la información de los diagnósticos, ampliando la especificidad de los códigos del CIE 10 para uso dentro de la institución.

En un futuro estudio se determinarán las circunstancias de los traumas oculares, de manera específica, con el 
fin de diseñar estrategias preventivas que ayuden a disminuir su frecuencia. Preferiblemente, el estudio contará con un diseño prospectivo para tener mejor recolección de los datos de diversas variables adicionales (por ejemplo: mecanismo del trauma, uso o no de elementos de protección, etc.).

\section{Conflicto de intereses}

Ninguno de los autores presenta conflicto de intereses con respecto a este artículo.

\section{Financiación}

Este trabajo no recibió financiación de ninguna entidad pública o privada.

\section{Referencias}

1. Girard B, Bourcier F, Agdabede I, Laroche L. [Activity and epidemiology in an ophthalmological emergency center]. J Fr Ophtalmol. 2002;25:701-11.

2. Salti HI, Mehanna CJ, Abiad B, Ghazi N, Raad S, Barikian A, et al. Changing trends in eye-related complaints presenting to the emergency department in Beirut, Lebanon, over 15 years. J Ophthalmol. 2018;2018:4739865

3. Leal MA, Beneyto P, Ibáñez MA, García A, Fernández MJ. ¿Han cambiado los pacientes oftalmológicos que acuden a urgencias? Estudio evolutivo entre los años 1997 y 2005. Arch Soc Esp Oftalmol. 2007;82:159-65.

4. Kumar NL, Black D, McClellan K. Daytime presentations to a metropolitan ophthalmic emergency department. Clin Experiment Ophthalmol. 2005;33:586-92.

5. Channa R, Zafar SN, Canner JK, Haring RS, Schneider EB, Friedman DS. Epidemiology of eyerelated emergency department visits. JAMA Ophthalmol. 2016;134:312-9

6. Sen E, Celik S, Inanc M, Elgin U, Ozyurt B, Y1lmazbas P. Seasonal distribution of ocular conditions treated at the emergency room: a 1-year prospective study. Arq Bras Oftalmol. 2018;81:116-9.

7. Jafari AK, Bozorgui S, Shahverdi N, Ameri A, Akbari MR, Salmasian H. Different causes of referral to ophthalmology emergency room. J Emerg Trauma Shock. 2012;5:16-22.

8. Baig R, Ahmad K, Zafar S, Khan NU, Ashfaq A. Frequency of ocular emergencies in a tertiary care setting in Karachi, Pakistan - It is time to reduce unnecessary visits. J Pak Med Assoc. 2018;68:1493-5.
9. Samoilă O, Ostriceanu S, Samoilă L. Epidemiology of ocular emergencies in Cluj ophthalmology clinic. Rom J Ophthalmol. 2016;60:165-9.

10. Domínguez-Serrano FB, Molina-Solana P, InfanteCossío M, Sala-Turrens J, Seva-Silva N, Rodríguez-de-la-Rúa-Franch E. Ophthalmological emergencies. An epidemiological study: Are resources been used properly? Arch Soc Esp Oftalmol. 2019;94:211-7.

11. Siempis T. Urgent Eye Care in the UK Increased demand and challenges for the future. Med Hypothesis Discov Innov Ophthalmol. 2014;3:103-10.

12. Perumal D, Niederer R, Raynel S, McGhee CN. Patterns of ophthalmic referral and emergency presentations to an acute tertiary eye service in New Zealand. N Z Med J. 2011;124:35-47.

13. Stagg BC, Shah MM, Talwar N, Padovani-Claudio DA, Woodward MA, Stein JD. Factors affecting visits to the emergency department for urgent and non-urgent ocular conditions. Ophthalmology 2017;124:720-9.

14. Edwards RS. Ophthalmic emergencies in a district general hospital casualty department. $\mathrm{Br} \mathrm{J}$ Ophthalmol. 1987;71:938-42.

15. Vieira GM. [One month in an eye emergency clinic in Brasilia].[Article in Portuguese]. Arq Bras Oftalmol. 2007;70:797-802.

16. Agrinier N, Conart JB, Baudot A, Ameloot F, Angioi-Duprez K. [Epidemiology of patients with eye-related emergencies in a university hospital: A cross-sectional study identifying factors associated with true emergency]. J Fr Ophtalmol. 2018 Jun;41(6):546-53.

17. Alabbasi OM, Al-Barry M, Albasri RF, Khashim HF, Aloufi MM, Abdulaal MF, et al. Patterns of ophthalmic emergencies presenting to a referral hospital in Medina City, Saudi Arabia. Saudi J Ophthalmol. 2017;31:243-6.

18. León F, Taboada JF, Guimerá V. Traumatismos Oculares graves en España: Factores epidemiológicos, estudio de las lesiones y medidas de prevención., Barcelona: Vallirana; 1994. p. 1227.

19. Fea A, Bosone A, Rolle T, Grignolo FM. Eye injuries in an Italian urban population: report of 10,620 cases admitted to an eye emergency department in Torino. Graefes Arch Clin Exp Ophthalmol. 2008;246:175-9.

20. Ong HS, Barsam A, Morris OC, Siriwardena D, Verma S. A survey of ocular sports trauma and the role of eye protection. Cont Lens Anterior Eye. 2012;35:285-7. 